\title{
Actin Dynamics in Growth Cones
}

\author{
Shigeo Okabe and Nobutaka Hirokawa \\ Department of Anatomy and Cell Biology, School of Medicine, University of Tokyo, Hongo, Tokyo, 113 Japan
}

The mechanism of actin incorporation and turnover in the nerve growth cone was examined by immunoelectron microscopy and low-light-level video microscopy of cultured neurons injected with biotin-labeled actin or fluorescently labeled actin.

We first determined the sites of actin incorporation into the cytoskeleton of growth cones by immunoelectron microscopy of cultured neurons injected with biotin-labeled actin and reacted with an anti-biotin antibody and goldlabeled secondary antibody. Shortly after the injection, biotin-actin molecules incorporated into the cytoskeleton were localized in the distal part of actin bundles in the filopodia and at the membrane-associated fringe of the actin filament network. With longer incubation, most actin polymers in the growth cones were labeled uniformly, suggesting that actin subunits are added preferentially at the membrane-associated ends of preexisting actin filaments.

We then determined whether actin filaments translocate within the growth cones by low-light-level video microscopy of living neurons injected with fluorescently labeled actin and photobleached with a laser beam. When actin fluorescence at the leading edge of a growth cone was bleached, a rearward translocation of the bleached spot toward the base of the growth cone was observed. This observation suggests the presence of a rearward flow of actin polymers within growth cones.

Taken together, these results indicate that there is a continuous addition of actin monomers at the leading edge of the growth cone and a successive rearward translocation of the assembled filaments.

The projection of axons to their appropriate target site is an important step in the generation of the neuronal network. The cytoskeleton provides important structural bases for this process. To understand the roles of the cytoskeleton in neuronal morphogenesis, we have been investigating the molecular architecture and dynamics of neuronal cytoskeletons. Neurofilaments and microtubules together with various kinds of microtubule-associated proteins form major dynamic frameworks in the shaft of the axon (Hirokawa, 1982, 1991; Hirokawa et al., 1985; Matus, 1988; Okabe and Hirokawa, 1988). On the other

\footnotetext{
Received Nov. 9, 1990; revised Jan. 28, 1991; accepted Jan. 31, 1991.

This work was supported by a special Grant-in-aid for Scientific Research from the Japan Ministry of Education, Science, and Culture, a grant from the Institute of Physical and (hemical Research (RIKEN), and a grant from the Naito Memorial Science Foundation to N.H.

Correspondence should be addressed to Shigeo Okabe, Department of Anatomy and Cell Biology, School of Medicine, University of Tokyo, 7-3-1, Hongo, Bunkyoku, Tokyo, 113. Japan.

Copyright (C) 1991 Society for Neuroscience $0270-6474 / 91 / 111918-12 \$ 03.00 / 0$
}

hand, motile endings of growing axons, known as growth cones, are thought to be important in causing and directing axon elongation (Goodman et al., 1985; Kater and Letourneau, 1985). The mechanism of axonal elongation and the role of growth cones in this event have been studied extensively (Marsh and Letourneau, 1984; Bray, 1987; Dennerll et al., 1988; Lamoureux et al., 1989). Studies of the growing neurons in culture have shown that the peripheral cytoplasmic region of growth cones is highly dynamic and undergoes cyclic movements of protrusion and retraction (Nakai and Kawasaki, 1960; Nakai, 1979; Bray and Chapman, 1985; Forscher et al., 1987). Two distinct types of protrusions can be observed in this peripheral domain: the filopodium (also called the microspike) and the lamellipodium. Both structures contain densely packed actin filaments (Letourneau, 1983; Bridgman and Dailey, 1989). In filopodia, actin filaments are tightly bundled. On the other hand, lamellipodia are filled with randomly oriented networks of actin filaments. Because the cytoskeleton is a major intrinsic determinant of the cell shape and is also involved in the generation of mechanical forces within the cytoplasm, information about the turnover of actin filaments in the growth cone will be required in order to attain an understanding of the molecular mechanism of growth cone motility and neurite elongation (Dennerll et al., 1988; Mitchison and Kirschner, 1988; Smith, 1988).

Actin filaments in the peripheral region of motile cells are highly dynamic. Actin turnover is well characterized in the leading edge of fibroblastic cells (Wang, 1985; Fisher et al., 1988). In our previous report, we showed that actin monomers are rapidly incorporated into the membrane-associated fringe of the actin filament network in fibroblasts (Okabe and Hirokawa, $1989 b$ ). Once actin filaments are assembled, they are supposed to be transported rearward from the leading edge. Wang (1985) has presented evidence that a centripetal flow of actin filaments occurs in the lamella of fibroblasts. In the case of the growth cone, however, there have been few experiments to reveal the basic mechanism of actin turnover. Recently, Forscher and Smith (1988) have documented the presence of a centripetal flow of the cytoplasm in the growth cones of Aplysia bag cell neurons. The effects of cytochalasins on this movement have suggested that the observed cytoplasmic density would be mainly composed of actin filaments.

The recently developed technique of microinjection of cytoskeletal proteins has enabled us to visualize directly the dynamics of the cytoskeletal system in vivo (Taylor and Wang, 1978; Kreis et al., 1979). This method has also been applied to nerve cells in culture, and information about the turnover of microtubules and actin filaments in the neurite shaft has been obtained (Okabe and Hirokawa, 1988, 1989a,b, 1990; Lim et al., 1989, 1990). In the present study, we examined the turnover of actin in growth cones by the method of microinjection of fluorescently 
labeled or biotin-labeled actin, and the results revealed the addition of subunits at the membrane-associated ends of the actin filament networks and retrograde translocation of assembled filaments within the growth cone.

\section{Materials and Methods}

Preparation of biotin-labeled and fluorescein-labeled actin. Biotin-labeled actin was prepared according to the method described previously (Okabe and Hirokawa, 1989b). Derivatization of actin with 5(6)carboxyfluorescein succinimidyl ester (Molecular Probes, Junction City, OR) was done according to the method of Kellog et al. (1988).

Cell culture. PC1 2 cells were grown at $37^{\circ} \mathrm{C}$ in Dulbecco's modified Eagle's medium (Gibco, Grand Island, NY) supplemented with 5\% precolostrum newborn calf serum (Mitsubishi Chemical Industries, Tokyo, Japan) and 5\% horse serum (Gibco; Hatanaka, 1981). The methods of cell fusion with polyethylene glycol and differentiation with NGF were described previously (Okabe and Hirokawa, 1988). Dorsal root ganglion (DRG) neurons were isolated from adult mice according to the method that had been originally developed for rabbit DRG neurons (Goldenberg and De Boni, 1983). The isolated DRG neurons were plated onto laminin-coated glass coverslips or 35- $\mathrm{mm}$ Petri dishes, which were prepared by the method of Rogers et al. (1983). Mouse 3T3 fibroblasts were cultured as previously described (Okabe and Hirokawa, 1989b).

Microinjection. Microinjection was performed according to the method described previously (Okabe and Hirokawa, 1988, 1989b). Biotinactin and fluorescein-actin were diluted to $2 \mathrm{mg} / \mathrm{ml}$ and $3-8 \mathrm{mg} / \mathrm{ml}$, respectively, before use, clarified by centrifugation with a Beckman TL100 ultracentrifuge, and loaded into necdles.

Immunofluorescence. Cells were briefly washed in $60 \mathrm{~mm}$ PIPES, 25 mM HEPES, $10 \mathrm{~mm}$ EGTA, and $2 \mathrm{~mm} \mathrm{MgCl}_{2}$ (pH, 6.9; PHEM buffer), lysed with PHEM buffer plus $0.3 \%$ Triton X-100 and $10 \mu \mathrm{M}$ Taxol for 2 min (for PC12 cells) or with PHEM buffer plus $0.02 \%$ saponin and $10 \mu \mathrm{M}$ Taxol for $4 \mathrm{~min}$ (for DRG neurons), and then fixed with $2 \%$ paraformaldehyde plus $0.1 \%$ glutaraldehyde in PHEM buffer for $30 \mathrm{~min}$. Procedures for immunofluorescence and phalloidin labeling were the same as described previously (Okabe and Hirokawa, 1989b).

Immunoelectron microscopy. The extraction and fixation procedures were the same as those for immunofluorescence except that $0.3 \%$ glutaraldehyde was used for fixation. The procedures of antibody labeling, postfixation, embedding, and sectioning were described previously (Okabe and Hirokawa, 1989b).

Low-light-level video microscopy. IIoles with a $30-\mathrm{mm}$ diameter were punched into Teflon holders, and glass coverslips with living neurons injected with fluorescein-actin were attached to them. To prevent evaporation of the culture medium, mineral oil was overlain. The culture chamber was placed on a microscope stage that was maintained at 35 $\pm 1^{\circ} \mathrm{C}$ with a hand-made air-curtain incubator.

Cells were illuminated intermittently with a halogen lamp (50 W) operated at $5-10 \mathrm{~V}$ and observed with a $100 \times$ fluor objective lens $(1.25$ NA; Nikon, Tokyo, Japan) on an IMT-2 inverted microscope (Olympus, Tokyo, Japan). Fluorescence images were recorded using a silicon-intensified target (SIT) camera (C2400-08, Hamamatsu Photonics, Hamamatsu, Japan) or an image intensifier coupled with a CCD camera (Hamamatsu Photonics, C2400-87; Okabe and Hirokawa, 1990). Video frames were summed and averaged for $0.5 \mathrm{sec}$, and background fluorescence was subtracted with a digital image processor (Hamamatsu Photonics, ARGUS-100). In addition, images were fed through the image processor and stored on tape using an SP.U-matic video cassette recorder (SONY, model VO-9600).

Photobleaching with a laser beam. The photobleaching apparatus was designed and assembled according to the method described previously (Hamaguchi et al., 1987; Okabe and Hirokawa, 1990). An argon ion laser was operated at 3-5 mW. An electronically controlled shutter was used to control precisely the exposure time $(8 \mathrm{msec})$.

Image analysis. Digitized images of fluorescence microscopy were quantitated with an image processor (ARGUS-100, Hamamatsu Photonics). We first set a rectangle along the course of the movement of a bleached spot. The average of fluorescence intensities across each row of pixels perpendicular to the long axis of the rectangle was calculated and plotted against the distance from the left side of the rectangle. Because each rectangle in a series of digitized images was set on a fixed position relative to the substratum, the observed movements of troughs on fluorescence intensity profiles were also relative to the substratum.

Other methods. Rhodamine-labeled BSA was prepared as follows: 5 $\mathrm{mg} / \mathrm{ml}$ of BSA in $10 \mathrm{~mm}$ HEPES $(\mathrm{pH}, 8.5)$ was reacted with $0.5 \mathrm{mg} / \mathrm{ml}$ 5(6)carboxytetramethylrhodamine succinimidyl ester (Molecular Probes, Junction City, OR) for $30 \mathrm{~min}$ at $24^{\circ} \mathrm{C}$, and the reaction was stopped by the addition of K-glutamate to the final concentration of $50 \mathrm{~mm}$. BSA and unbound dye were separated on a Sephadex G-25 column, and rhodamine-BSA-containing fractions were collected and concentrated by precipitation with $70 \%$ saturated ammonium sulfate. The pellet was dissolved in $50 \mathrm{mM} \mathrm{K}$-glutamate and $0.5 \mathrm{mM} \mathrm{MgCl}_{2}(\mathrm{pH}$, 6.8). SDS-gel electrophoresis was performed according to the method of Laemmli (1970).

\section{Results}

Growth cone morphology

Growth cones of PC12 cells plated onto poly-L-lysine-coated substrate were usually 5-10 $\mu \mathrm{m}$ wide and had 2-20 filopodia. Although large lamellipodia were rarely observed at their leading edge, electron microscopic observations revealed that small sheetlike veils always existed between any two filopodia (data not shown).

DRG neurons regenerated their neurites rapidly after plating. The neurites were tipped by large growth cones ( $10-40 \mu \mathrm{m}$ wide), which were highly motile and had distinct lamellipodia along their periphery.

These two types of growth cones were analyzed by microinjection of biotin-actin and immunoelectron microscopy with an anti-biotin antibody. Photobleaching experiments were carried out with the growth cones of DRG neurons, because their large, flat shape was more suitable for analysis on a light microscopic level.

\section{Incorporation of biotin-actin into the growth cone cytoskeleton}

To analyze the actin dynamics in nerve growth cones, we first determined the sites of actin incorporation into the cytoskeleton of growth cones by using the method of microinjection of biotinlabeled actin into cultured neurons, followed by successive immunocytochemistry of the injected cells with an anti-biotin antibody. The biotin-actin preparations have previously been shown to behave in the same manner as native actin in vitro and to be incorporated into all actin-containing structures of fibroblasts (Okabe and Hirokawa, 1989b). We used PC12 rat pheochromocytoma cell lines and a primary culture of mouse DRG neurons for the microinjection. Figure 1 shows the growth cones of a PC12 cell and a DRG neuron incubated for $>40 \mathrm{~min}$ after injection. The pattern of staining with antibiotin is almost identical to that of rhodamine-phalloidin, which reveals the distribution of total actin filaments in growth cones. This result indicates that biotin-actin has the ability to be incorporated into the native cytoskeleton of growth cones.

To determine the sites of actin incorporation into the cytoskeleton of growth cones, eight PC12 cells and seven DRG neurons injected with biotin-actin were analyzed with an electron microscope. Incubation time after injection ranged from 4 to $68 \mathrm{~min}$ for PC12 cells and from 2.5 to $39 \mathrm{~min}$ for DRG neurons. Through the course of this analysis, we found that the time course of biotin-actin incorporation differs between proximal growth cones and distal ones. In proximal growth cones (within $50 \mu \mathrm{m}$ from cell bodies), biotin-actin was already incorporated into the cytoskeleton within $5 \mathrm{~min}$ after injection. In contrast, incorporation of biotin-actin was not observed in distal growth cones ( $>200 \mu \mathrm{m}$ away from cell bodies) at this time point. Because it takes a certain time for injected molecules to reach distal growth cones, this difference would be due to the time lag of the entry of injected molecules into individual growth cones. 

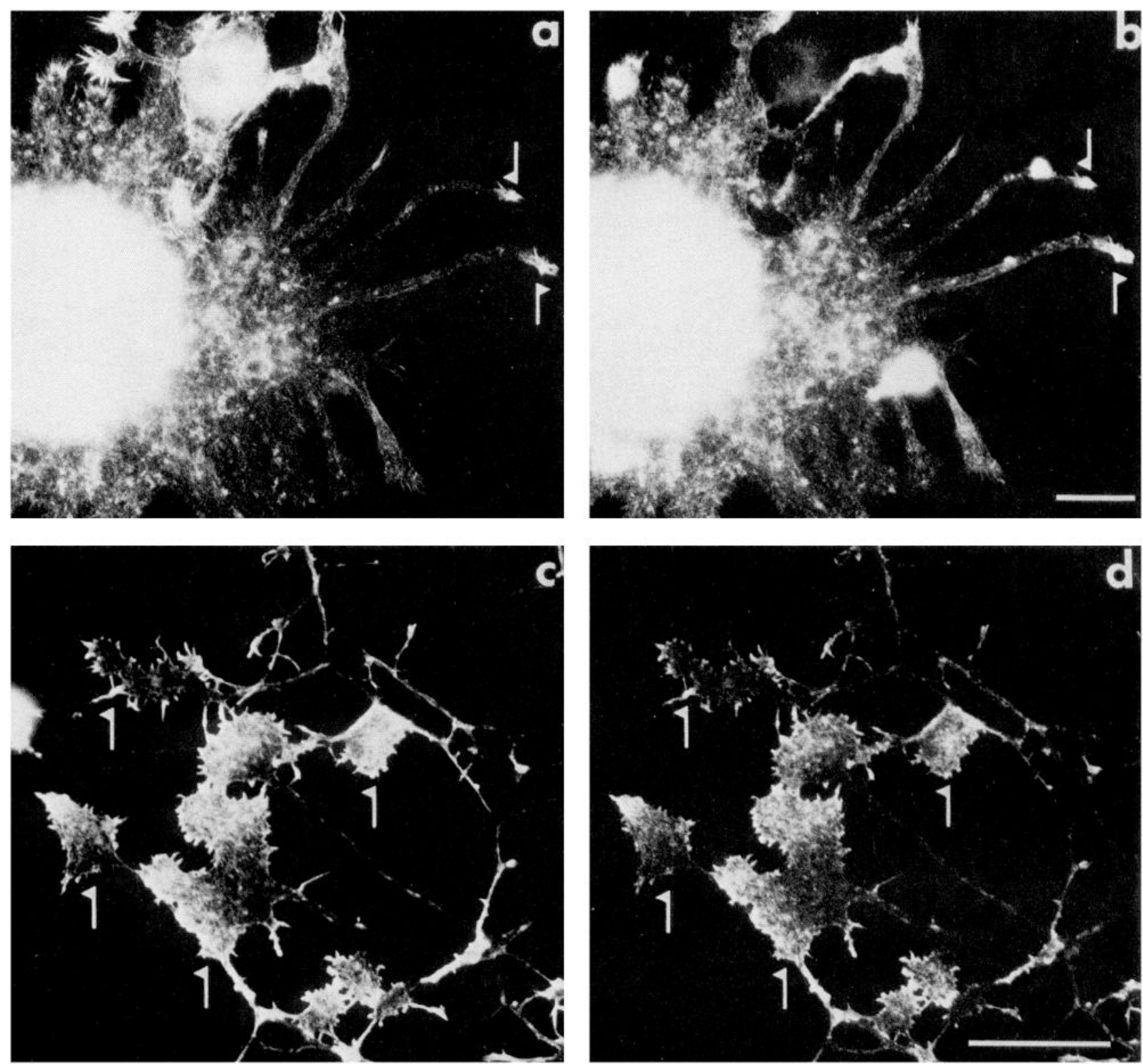

Figure 1. Incorporation of biotin-actin into the cytoskeleton of PC12 cells and DRG neurons. When cells were incubated for $>40$ min after injection, anti-biotin staining of growth cones (arrows in $b, d$ ) was almost identical to the staining of rhodamine-phalloidin (arrows in $a$, $c$ ). $a$, Rhodamine-phalloidin staining of a PC12 cell permeabilized 40 min after injection. $b$, Anti-biotin staining of the same cell as in $a$. $c$, Rhodaminephalloidin staining of growth cones of a DRG neuron permeabilized $48 \mathrm{~min}$ after injection. $d$, Anti-biotin staining of the same cell as in $c$. Scale bars, $20 \mu \mathrm{m}$.

At 7-15 min after injection, growth cones $>50 \mu$ m away from cell bodies began to incorporate biotin-actin, and biotin-actin incorporation at the distal part of the growth cone cytoskeleton was frequently observed. Figure $2 a$ shows a growth cone of a PC1 2 cell permeabilized $8 \mathrm{~min} 1 \mathrm{sec}$ after the injection. The biotin-actin assembled after the injection was recognized by an antibody to biotin and a gold-labeled secondary antibody. Gold particles were mainly localized in the distal part of actin bundles (Fig. 2a, arrows) and the membrane-associated fringe of the actin filament network (arrowheads). A similar pattern of incorporation of biotin-actin was observed in the growth cones of DRG neurons. Figure 3, $a$ and $b$, shows growth cones of a cell permeabilized $9 \min 41 \mathrm{sec}$ after the injection. Gold particles were localized at the membrane-associated fringe of the actin filament network. The polarized pattern of biotin-actin incorporation at the leading edge was consistently observed in the distal growth cones of two PC12 cells (permeabilized and fixed $8 \mathrm{~min} 1 \mathrm{sec}$ and $10 \mathrm{~min} 25 \mathrm{sec}$ after injection) and two DRG neurons (permeabilized and fixed $7 \mathrm{~min} 32 \mathrm{sec}$ and $9 \mathrm{~min} 41$ sec after injection). We analyzed $>10$ growth cones for each injected cell and found that about one-third of distal growth cones were significantly labeled with gold-conjugated antibodies. These growth cones incorporated biotin-actin exclusively at the leading edge (Figs. $2 a ; 3 a, b$ ). In contrast, the remaining two-thirds contained few gold particles, suggesting that the injected molecules had not yet reached these growth cones at the time of fixation.

As incubation time lengthened, more gold particles were de- 

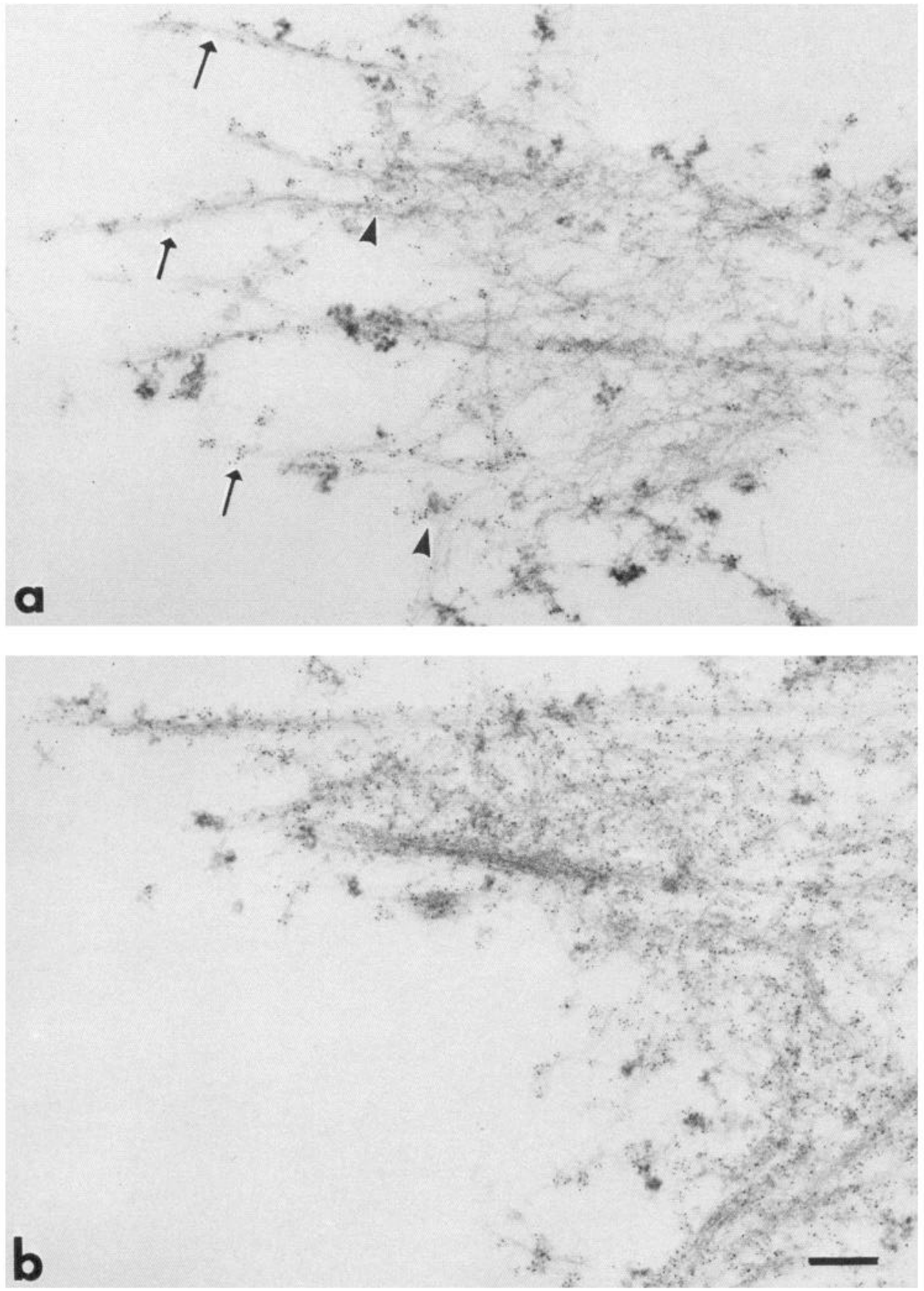

Figure 2. Time course of biotin-actin incorporation into the actin filament network of growth cones of PC12 cells. Growth cones of PC12 cells were permeabilized and fixed at different time points after injection. Labeled actin subunits assembled after injection were recognized by an antibody to biotin and a gold-labeled secondary antibody. $a, \mathrm{~A}$ growth cone of a $\mathrm{PC} 12$ cell permeabilized $8 \mathrm{~min} 1 \mathrm{sec}$ after injection. Biotinactin molecules were localized in the distal part of actin bundles (arrows) and the distal fringe of the actin filament network in the growth cone (arrowheads). $b, \mathrm{~A}$ growth cone of a PC12 cell incubated for $1 \mathrm{hr}$ after injection. Uniform incorporation of biotin-actin was observed. Scale bar, $200 \mathrm{~nm}$. tected in distal growth cones, and the polarized pattern of incorporation was less frequently observed. By $40 \mathrm{~min}$ after injection, most actin filaments in the growth cones were labeled uniformly (Figs. $2 b$ for PC1 2 cells; $3 c$, $d$ for DRG neurons). Time course of biotin-actin incorporation did not differ significantly between PC12 cells and DRG neurons. These results indicate that the assembly of actin molecules occurs at the distal part of actin bundles and the membrane-associated fringe of the actin filament network in the growth cone.

\section{Retrograde translocation of actin filaments within growth cones}

To further characterize the mechanism of actin turnover, we analyzed the recovery of fluorescence after photobleaching of a fluorescent analog of actin in the growth cone of DRG neurons. Figure 4 shows $N$-hydroxysuccinimidyl fluorescein-conjugated actin subjected to electrophoresis in a $10 \%$ SDS polyacrylamide gel. This preparation was free of other contaminating proteins or degradation products (Fig. 4, lane 2). Figure 4, lane 3, also shows that only the actin band was fluorescent and that the removal of unconjugated dye was complete. We compared the viscometric properties of unlabeled and fluorescein-conjugated actin. As shown in Figure 5, both unlabeled and labeled actin displayed similar viscosities, and the estimated critical concentrations were almost identical. When this fluorescent probe was injected into fibroblastic cells, it was incorporated into actincontaining cytoplasmic structures such as stress fibers and ruffling membranes (Fig. 6). These results indicate that $N$-hydroxy- 

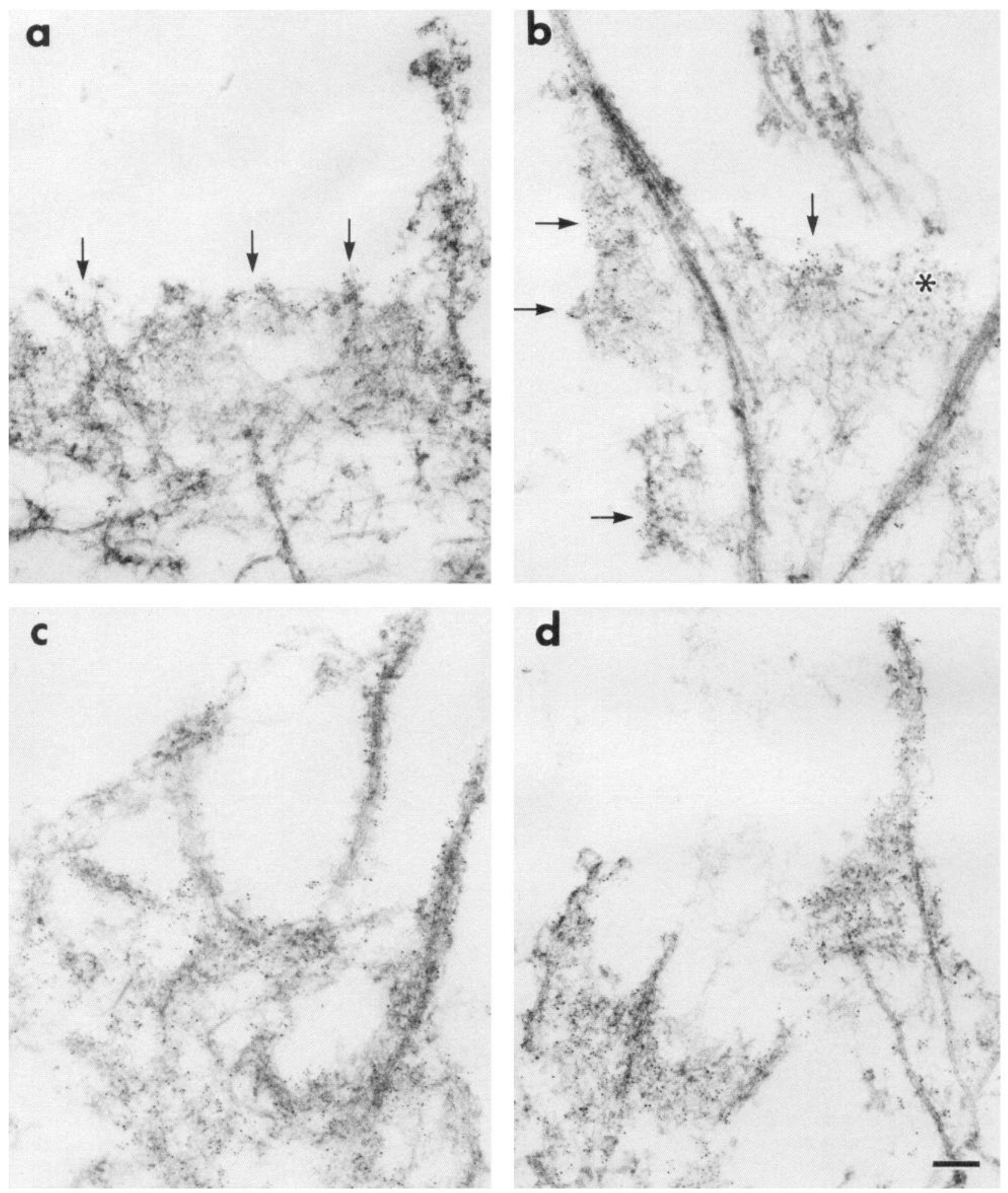

Figure 3. Growth cones of DRG neurons injected with biotin-actin. Biotin-actin assembled after injection was recognized by an antibody to biotin and a gold-labeled secondary antibody. $a$ and $b$, Growth cones of a DRG neuron permeabilized 9 min 41 sec after injection. Biotin-actin molecules were localized at the membrane-associated fringe of the actin filament network in lamellipodia (arrows). Asterisk, extracellular matrix. $c$ and $d$, Growth cones of a DRG neuron permeabilized $39 \mathrm{~min}$ after injection. Actin filament bundles and networks in filopodia $(c)$ and lamellipodia (d) were labeled uniformly, suggesting that the injected biotin-actin was fully incorporated at this time point. Scale bar, $200 \mathrm{~nm}$ for $a, 150 \mathrm{~nm}$ for $b-d$. 


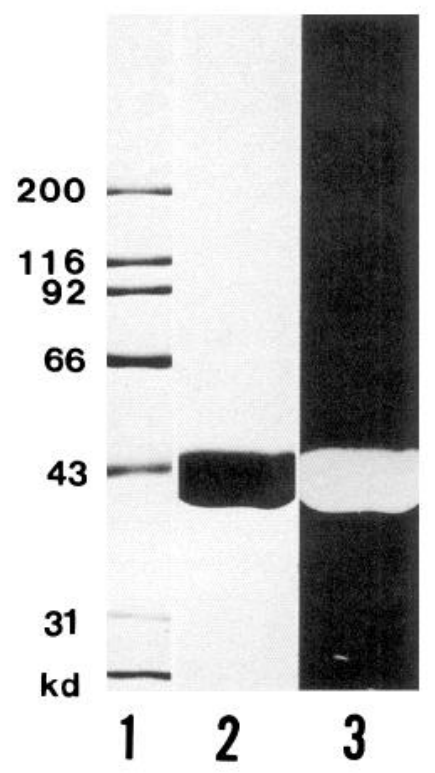

Figure 4. SDS-PAGE analysis of the preparation of fluorescein-labeled actin. Actin prepared from chicken breast muscle was labeled with $\mathrm{N}$-hydroxysuccinimidyl fluorescein. A single band with the same electrophoretic mobility as actin ( $43 \mathrm{kDa}$ ) is observed (lane 2), and the fluorescence photograph shows that only the actin band is fluorescent (lane 3). Lane 1, molecular weight standard.

succinimidyl fluorescein-conjugated actin can be used as a probe for actin dynamics in vivo.

DRG neurons with rapidly extending neurites were injected with fluorescein-labeled actin. After incubation for $2-4 \mathrm{hr}$ to allow for the uniform incorporation of injected molecules, a small region of a fluorescent growth cone was bleached with a laser beam, and fluorescent images were recorded intermittently. When the distal margin of a growth cone was bleached, we frequently observed a polarized pattern of fluorescence recovery as shown in Figure 7. Figure $7 a$ shows a fluorescent growth cone whose distal portion was bleached. Immediately after the bleaching, a bleached spot was clearly observed (Fig. $7 b$ ). The recovery of fluorescence was first observed at the distal fringe of the growth cone (Fig. $7 c$, arrow). As the fluorescence recovered at the distal edge, the bleached spot seemed to be translocated in a centripetal fashion (Fig. $7 b-d$ ). To confirm the possible translocation of the bleached spot, we created fluorescence intensity profiles from the digitized, processed fluorescence images (Fig. 8a-d). We first determined the center of each bleached spot (Fig. $8 c, d, X_{1}, X_{2}$ ) and calculated the distance between the two centers (Fig. $8 d, \Delta X$ ) as the translocation distance. The rate of rearward movement was then estimated to be $0.98 \mu \mathrm{m} / \mathrm{min}$. We observed substantial broadening of the bleached region between sequential fluorescence images, and it is possible that the observed translocation of the center of the bleached spot is an artifact of spot broadening. To test this possibility, we measured radii of bleached spots and calculated $\Delta R=R_{2}-R_{1}\left(R_{i}\right.$, radius of bleached spot at frame $i$ ). $\Delta R$ was always smaller than $\Delta X$ (in Fig. 8, $\Delta R$ was about half of $\Delta X$ ), suggesting that the translocation of the center of the bleached spot cannot be explained by the broadening of bleached spots.

Because the intensity profiles were plotted with respect to a fixed point on the substratum, the movement of the bleached spot was not relative to the front of the advancing growth cone,

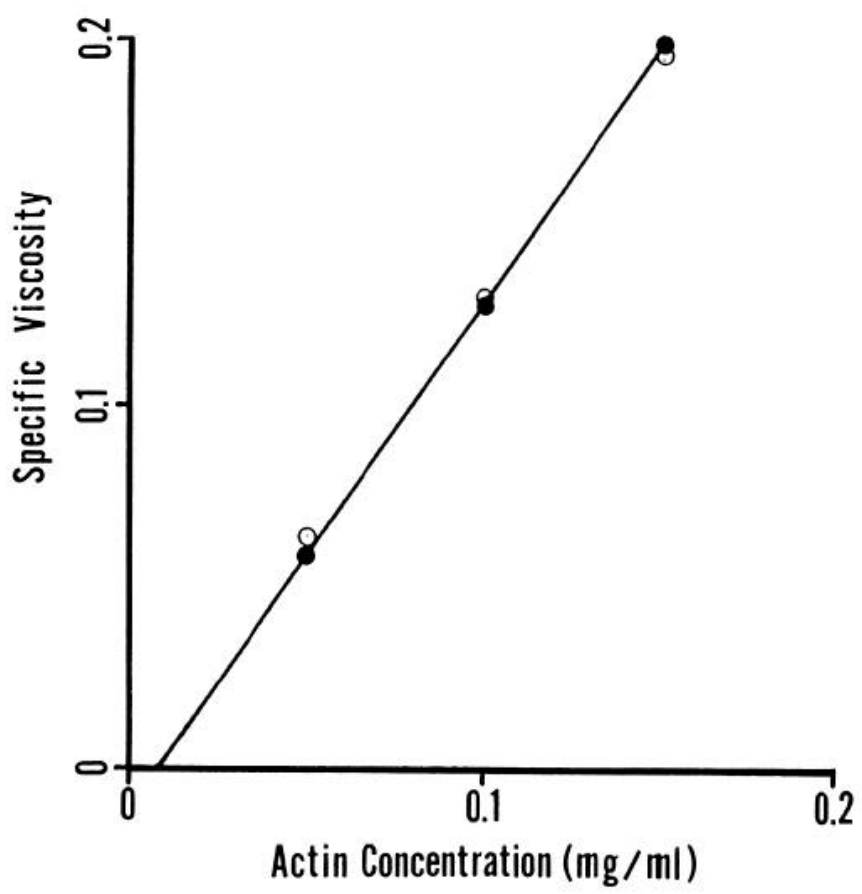

Figure 5. Ostwald capillary viscometry of labeled (0) and unlabeled $(\bullet)$ F-actin solutions. Monomeric actin was polymerized by the addition of (final concentrations) $50 \mathrm{mM} \mathrm{KCl}$ and $1 \mathrm{mM} \mathrm{MgCl}{ }_{2}$ and then incubated for $2 \mathrm{hr}$ at $25^{\circ} \mathrm{C}$. The solid line indicates the slope calculated from the unlabeled F-actin solutions.

but rather to the substratum. During the period between the two frames shown in Figure 7, $c$ and $d$, the leading edge of the growth cone also advanced (Fig. $8 c, d, \Delta F$ ). The intensity profiles show that the rate of advancement was much slower than the rate of rearward movement of the bleached spot.

From ten photobleaching experiments, the translocation rate of the bleached zones was calculated to be $1.57 \pm 0.46 \mu \mathrm{m} /$ $\mathrm{min}$. This value is in the same range of the transport rate of actin filaments in the peripheral regions of motile cells as determined previously by several different methods (Wang, 1985; Forscher et al., 1987; Forscher and Smith, 1988).

When long filopodia were bleached with a laser beam, the recovery of fluorescence also followed a highly polar pattern. It was first detected at the distal segment of a filopodium (Fig. $9 c$, arrow) and then was seen to move toward the proximal part (Fig. 9d, arrow). Figure 10 shows fluorescence intensity profiles of the four digitized images of Figure 9. The arrows in Figure 10 indicate the proximal end of the fluorescent segment of the bleached filopodium, and it is clear that the fluorescence elongated toward the center of the growth cone. The second peak from the right in Figure 9, $b-d$, also increased rapidly, but this peak reflects the fluorescence intensity of a new filopodium protruding perpendicularly to the bleached filopodium (Fig. $9 d$, arrowhead). Because the entire length of the fluorescent filopodium was bleached, the movement of the proximal edge of the bleached spot was not evident in this experimental run.

Table 1 summarizes the last 42 runs of the photobleaching experiments. We bleached the leading edge of growth cones in 32 of the 42 runs and the proximal region in the remaining runs; 24 of 32 growth cones bleached at their leading edge showed centripetal recovery of fluorescence. Eight of 32 growth cones bleached at their leading edge changed their shape rapidly and it was difficult to determine whether or not centripetal recovery 


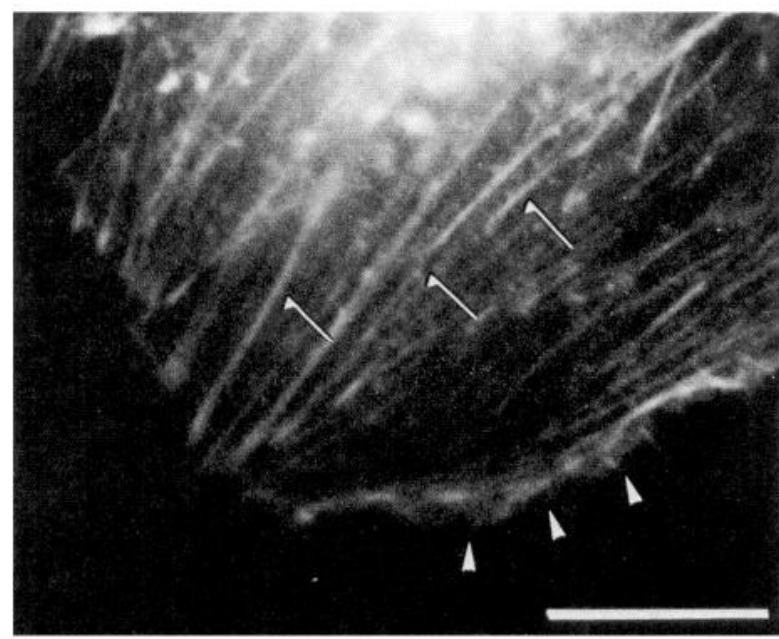

Figure 6. Low-light-level video microscopy of a living 3T3 fibroblast injected with fluorescein-labeled actin $4 \mathrm{hr}$ before observation. Fluorescein-labeled actin molecules are incorporated into actin-rich cytoplasmic structures such as stress fibers (arrows) and ruffling membrane (arrowheads). Scale bar, $10 \mu \mathrm{m}$.

occurred. At least the fluorescence of these eight growth cones never recovered in a proximodistal fashion. In 24 growth cones that showed centripetal recovery, 10 showed a net translocation of the bleached spots. The reason for the relatively large number (14 of 24) of growth cones without apparent net translocation is not clear. One possibility is that the diameter of the bleached spot (about $5 \mu \mathrm{m}$; see Fig. $8 b$ ) was relatively large and that the entire length of the actin-rich, peripheral region was bleached in these experiments (Fig. $9 b$ ). Otherwise, actin filaments might not translocate persistently, and the retrograde movement might occur at specific stages of growth cone advance. Further correlative studies on the translocation of bleached spots and mode of axon elongation should be necessary to determine this point. Of 10 growth cones bleached at their proximal region, eight showed centripetal recovery of fluorescence. However, the translocation of the bleached spot was never observed (data not shown).

Regardless of where a laser pulse was applied, the complete recovery of fluorescence was observed within $8 \mathrm{~min}$ after photobleaching. The observed turnover rate was much faster than that in the axon ( $>15 \mathrm{~min}$ for complete recovery), which had been determined by the same method (Okabe and Hirokawa, 1990).

To determine whether or not the observed pattern of fluorescence recovery primarily reflects the movement of actin polymers, we measured the rate of fluorescence recovery of freely mobile molecules such as rhodamine-conjugated BSA within growth cones. Fluorescence recovery was completed within 1 min after photobleaching, suggesting that the translocation of bleached spots over a period of several minutes is mainly due to the movement of actin polymers within growth cones (Fig. $11)$.

\section{Discussion}

The aim of the present study was to determine the basic mechanism of actin turnover in growth cones by using the technique of microinjection of biotin-labeled or fluorescein-labeled actin. Visualization of biotin-labeled actin incorporated into the cytoskeleton of growth cones by immunoelectron microscopy has revealed that the incorporation sites of monomeric actin are the membrane-associated ends of preexisting actin filament networks in the leading edge. Furthermore, photobleaching of the growth cones of cells injected with fluorescein-labeled actin presented evidence of the retrograde translocation of assembled actin filaments.

\section{Incorporation of monomeric actin into the growth cone cytoskeleton}

We observed rapid incorporation of biotin-actin at the membrane-associated peripheral regions of growth cones. A similar pattern of biotin-actin incorporation has been observed in the peripheral region of fibroblasts (Okabe and Hirokawa, 1989b). Thus, it is likely that the assembly of actin filaments in the peripheral region of motile cells is regulated by a common mechanism. Actin filaments in the leading edge of motile cells are known to be oriented with their barbed ends toward the cell periphery (Small et al., 1978). Because the barbed end is the preferred site of actin polymerization in vitro (Korn, 1982), it was postulated that it is also the preferred site of monomer addition in vivo. It is therefore probable that the observed pattern of actin incorporation results from the incorporation of actin monomers into the membrane-associated, namely, barbed ends of preexisting filaments at the leading edge. There is still another possibility, that is, that continuous de novo assembly of filaments occurs at the leading edge. However, it is difficult to differentiate between these possibilities because the arrangement of single actin filaments in growth cones could not be clearly observed in thin sections.

\section{Rearward translocation of assembled actin filaments}

There has been increasing evidence of a centripetal transport of some cellular components in the peripheral region of motile cells. The previously reported transport phenomena include actin filaments (Heath, 1981; Wang, 1985; Fisher et al., 1988; Forscher and Smith, 1988), membrane glycoproteins (Sheetz et al., 1989), membrane ruffles (Abercrombie et al., 1970), and

Table 1. Summary of photobleaching experiments

\begin{tabular}{|c|c|c|c|c|}
\hline \multirow{4}{*}{$\begin{array}{l}\text { Photobleaching } \\
\text { condition }\end{array}$} & \multicolumn{3}{|c|}{ Centripetal recovery of fluorescence } & \multirow[b]{4}{*}{ Total } \\
\hline & Positive & & Negative & \\
\hline & \multicolumn{2}{|c|}{$\begin{array}{l}\text { Net translocation of } \\
\text { bleached spot }\end{array}$} & & \\
\hline & Positive & Negative & & \\
\hline Photobleaching of the leading edge & 10 & 14 & 8 & 32 \\
\hline Photobleaching of the proximal region & 0 & 8 & 2 & 10 \\
\hline
\end{tabular}



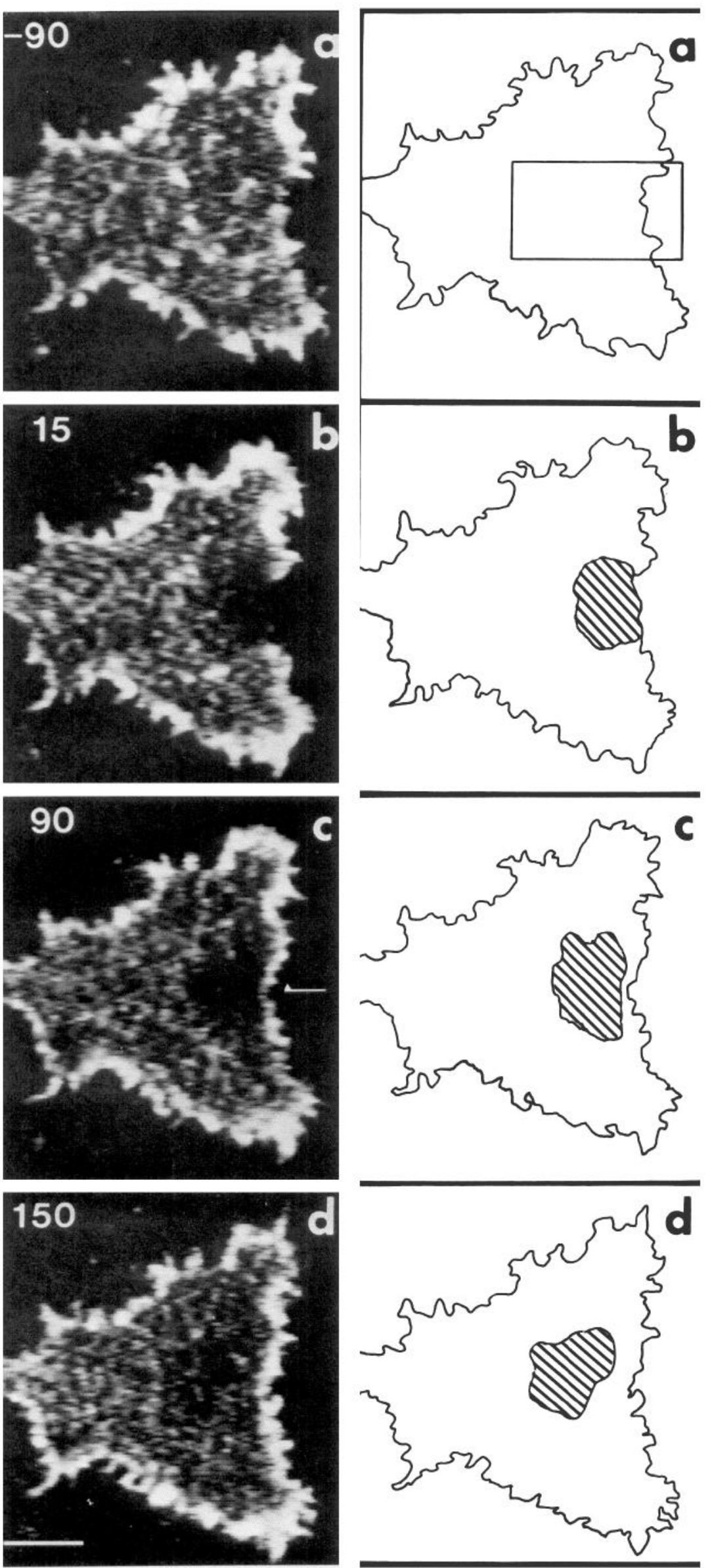

Figure 7. Fluorescence recovery after photobleaching of fluorescein-labeled actin in a growth cone of a DRG neuron. DRG neurons were microinjected with fluorescein-labeled actin 4-6 hr after plating, incubated for $2-4 \mathrm{hr}$, and then analyzed by low-light-level video microscopy. A distal region of a growth cone was bleached with a laser beam (b), and the process of recovery was observed intermittently. Elapsed time (in sec) after photobleaching is shown in the upper left corner of $a-d$. The recovery of fluorescence was first detected at the distal fringe (arrow in $c$ ) and was then seen to move toward the proximal region $(d)$. The translocation of the bleached spot itself toward the rear of the growth cone was also observed $(b-$ $d$ ). The right column shows tracings of video images, and bleached spots are represented by hatched areas. The rectangle in $a$ indicates the image area used for creating intensity profiles shown in Figure 8 . Scale bar, $5 \mu \mathrm{m}$. 


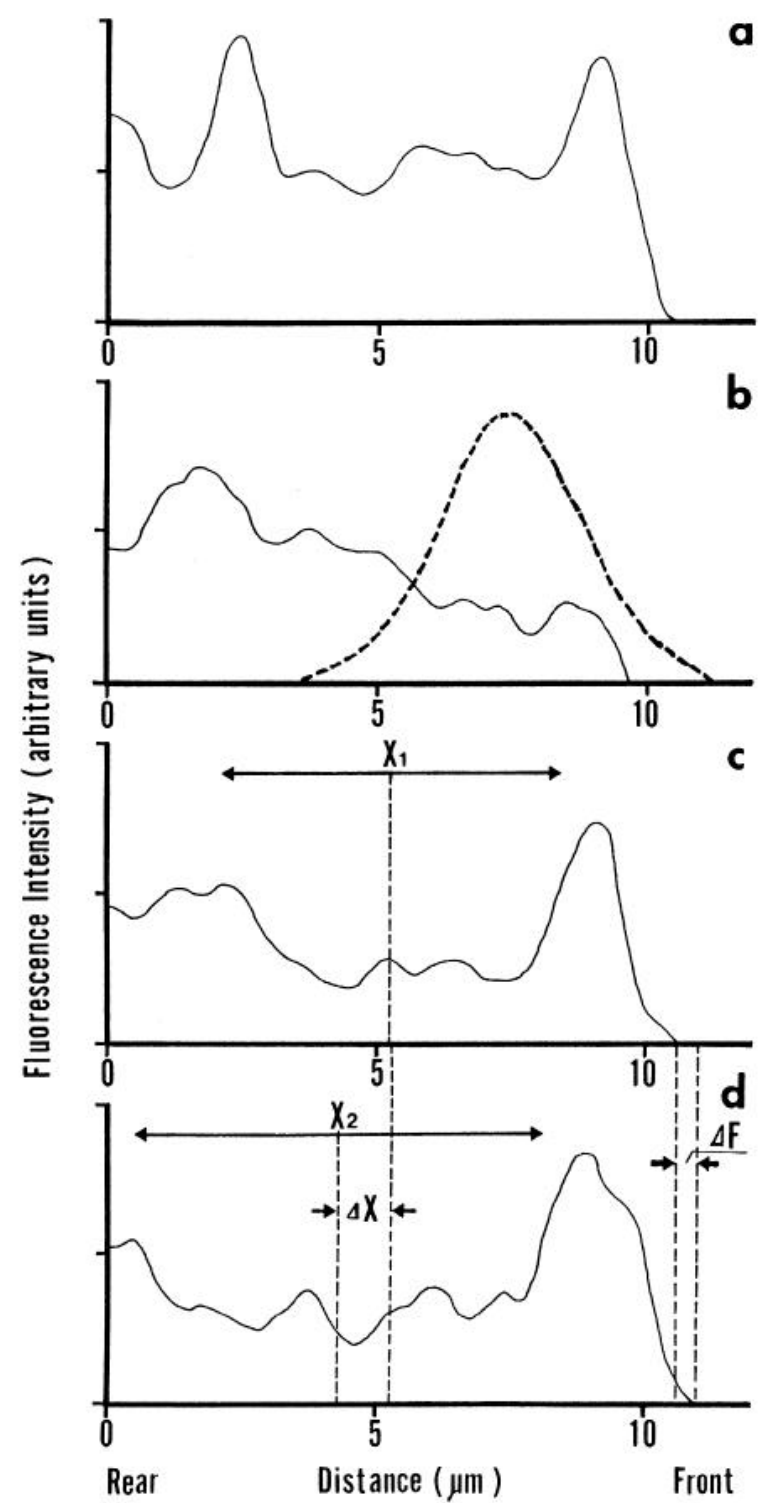

Figure 8 . The fluorescence intensity profiles corresponding to images $a-d$ of Figure 7. The ordinate shows the average of fluorescence intensities across each row of pixels perpendicular to the long axis of the rectangle shown in Figure $7 a$ (right). The abscissa shows the distance from the left side of the rectangle. Before bleaching, fluorescence intensity was high at the leading edge $(a)$. The broken line in $b$ indicates the intensity profile of the laser beam, and a marked decrease of fluorescence is observed at the leading edge. Fluorescence recovery was rapid at the distal fringe $(c)$, and the bleached zone was translocated in a centripetal fashion ( $X$, and $X$, in $c$ and $d$, respectively). During the observation, the net advance of the growth cone also occurred ( $\Delta F$ in $d)$, but it was less than the retrograde translocation of the bleached spot.

large particles adhering to the surface membrane (Harris and Dunn, 1972). It should be emphasized that the rates of transport of these cellular components are in the same range, suggesting that one mechanism of force generation could be responsible for the movements of all the components. In this sense, the actin filament system in combination with myosin ATPase is the most likely element to drive this complex system (Mitchison and Kirschner, 1988).

We have shown that the translocation of photobleaching spots at the leading edge occurs frequently in advancing growth cones.
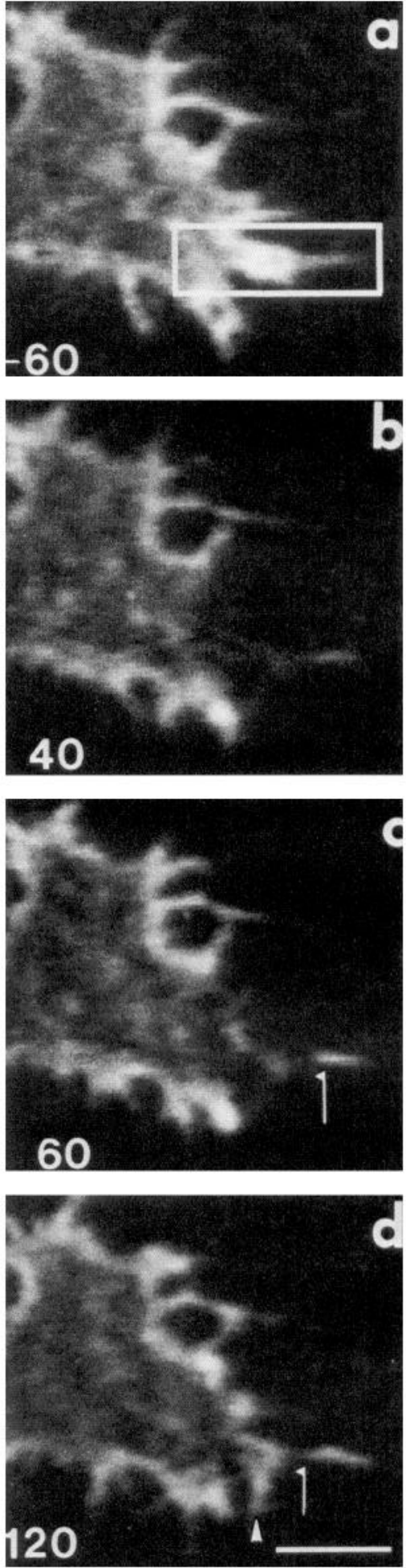

Figure 9. Fluorescence recovery after photobleaching of a long filopodium of a growth cone. The entire length of the filopodium was bleached with a laser beam $(b)$, and the process of recovery was observed. Elapsed time (in sec) after photobleaching is shown in the lower left corner of $a-d$. The recovery of fluorescence was first detected at the distal part of the long filopodium (arrow in $c$ ), which then moved toward the proximal part (arrow in $d$ ). The rectangle in $a$ indicates the image area used for creating intensity profiles shown in Figure 10. Scale bar, $5 \mu \mathrm{m}$. 


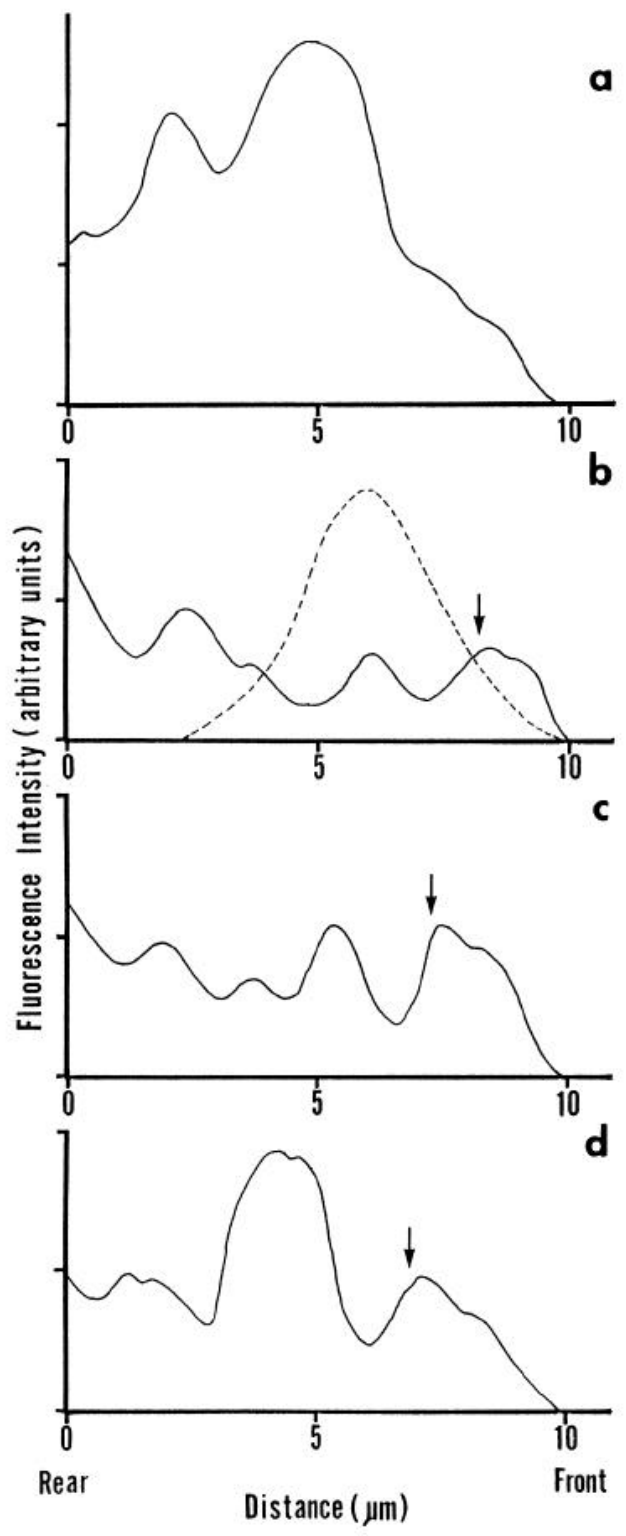

Figure 10. The fluorescence intensity profiles corresponding to images $a-d$ of Figure 9. The ordinate shows the average of the fluorescence intensities across each row of pixels perpendicular to the long axis of the rectangle shown in Figure $9 a$. The abscissa shows the distance from the left side of the rectangle. The broken line in $b$ indicates the intensity profile of the laser beam, and a marked decrease of fluorescence is observed at the leading edge $(b)$. After photobleaching, fluorescence intensity first increased at the distal segment of the filopodium (arrow in $c$ ), and this increase was propagated toward the rear of the growth cone (arrow in $d$ ).

One should note that the translocation of bleached spots is a process that continued over a period of several minutes. Because the photobleaching experiments of fluorescent, soluble proteins within growth cones suggested that the fluorescence recovery of monomeric actin would be completed within $1 \mathrm{~min}$ (Fig. 11), it is most likely that the observed translocation is due to the retrograde movement of actin filaments. Although several recent reports on the dynamics of actin filaments at the cell margin suggest that the rearward movement of actin filaments is an active, energy-dependent process (Mitchison and Kirschner, 1988; Smith, 1988), it is also possible that the observed trans-
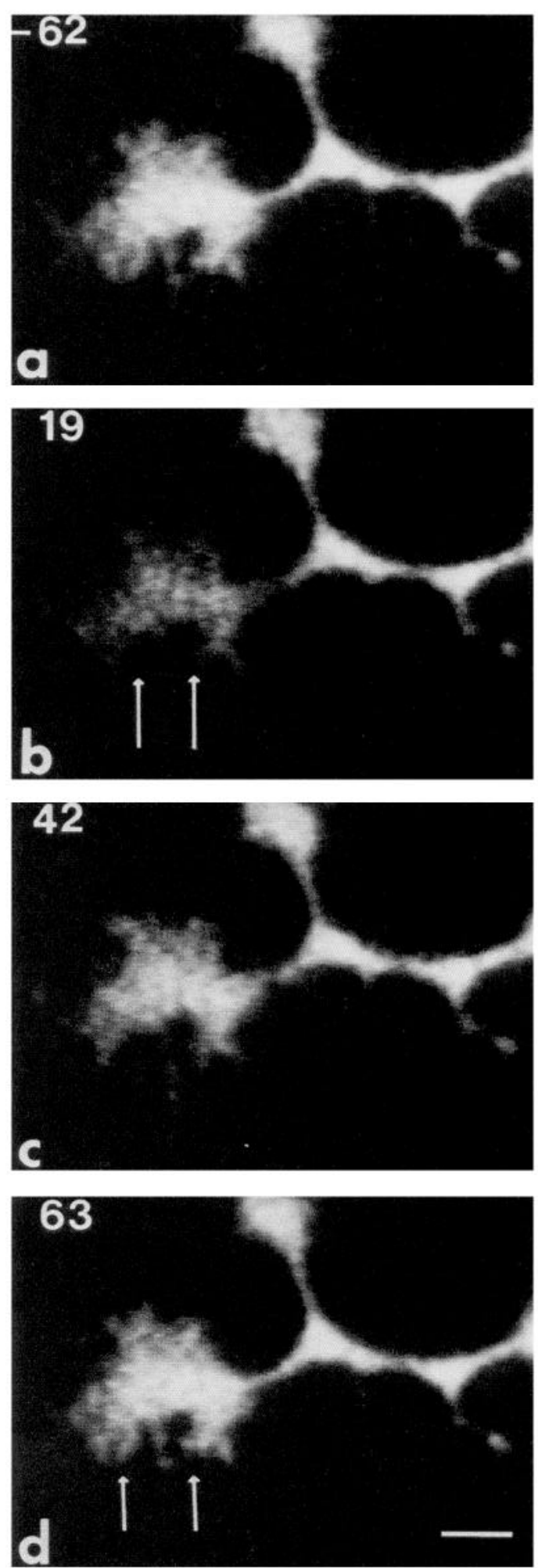

Figure 11. Fluorescence recovery after photobleaching of a growth cone of a DRG neuron injected with rhodamine-BSA. Due to the rapid movement of rhodamine-BSA molecules, a bleached spot could not be clearly observed immediately after bleaching, and the fluorescence intensity of the entire growth cone decreased (arrows in $b$ ). Fluorescence recovery was completed within $1 \mathrm{~min}$ after photobleaching (arrows in d). Elapsed time (in sec) after photobleaching is shown in the upper left corner of $a-d$. Scale bar, $5 \mu \mathrm{m}$. 


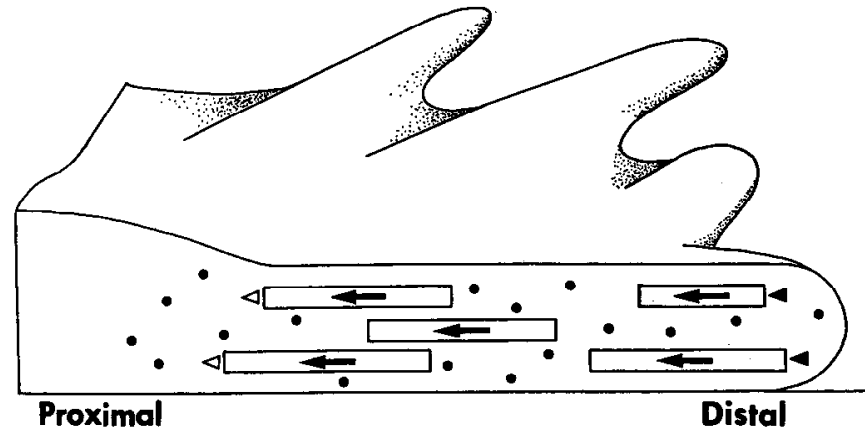

Figure 12. Model for actin turnover in the growth cone. Actin monomers (solid circles) are moved to the distal fringe of the growth cone and added to the barbed ends of preexisting actin filaments (solid arrowheads). The actin filaments are translocated toward the rear of the growth cone (arrows), where they are incorporated into the cortical actin networks of the neurite shaft or disassembled (open arrowheads).

location is driven by a complex of passive diffusion of actin filaments and addition of newly arrived monomers at the leading edge. To distinguish these possibilities, we measured radii of the bleached spots and compared the increase of radii with the translocation distance. The increase of radii was much smaller than the translocation distance, suggesting that the translocation of the bleached spots cannot be explained by the passive diffusion of filamentous actin. However, it should be noted that the broadening of the bleached spots was frequently observed in our experiments, and it is possible that our method of calculating the translocation distance might have overestimated the true translocation rate of actin filaments. Further quantitative studies would be needed to determine precisely the translocation rate of actin filaments in growth cones.

What is the biological significance of the retrograde actin flow in growth cones? Video analysis of growth cones has revealed that the protrusion of filopodia and lamellipodia is usually followed by their retraction, and that the net advance of the leading edge is much slower than the forward movement of the filopodia and lamellipodia (Nakai and Kawasaki, 1960; Bray and Chapman, 1985). These observations suggest that the protrusive and retractive movements of the leading edge are regulated by the balance between the retrograde movement of actin filaments and the assembly of actin at the plasma membrane. It is indeed possible that this balance is modulated locally by neurotrophic signals via second-messenger systems (Gunderson and Barretl, 1980 ) and that it would play an important role in the process of neuronal pathfinding.

\section{Actin turnover in growth cones}

The basic features of actin turnover in growth cones can be summarized as shown in Figure 12. Actin monomers are added to the barbed ends of preexisting actin filaments at the leading edge, and the assembled filaments are translocated toward the rear of the growth conc. When the filaments reach the rear, they would either be incorporated into the actin cytoskeleton at the base of the growth cone, or they would be disassembled to generate the monomeric actin pool. Our data indicate that the rate of rearward actin movement (about $1.5 \mu \mathrm{m} / \mathrm{min}$ ) is much faster than the rate of net translocation of the growth cone itself $(<0.3 \mu \mathrm{m} / \mathrm{min}$; see Fig. 8). This suggests that the amount of transported actin molecules is large and that an accumulation of actin filaments in the central region of the growth cone would be observed if there were no disassembly of filaments. However, the actin concentration is lower in the central region of the growth cone, strongly suggesting the presence of a mechanism that selectively disassembles actin filaments reaching the rear of the growth cone (Bamburg and Bray, 1987). By simple diffusion or by some other mechanism, actin monomers would be transported to the leading edge again and incorporated into the cytoskeleton. Although these last two steps are still conjecture and not based on experimental data, such a local actin cycle would be necessary in order to explain the observed actin incorporation and translocation.

What kinds of actin-activated ATPase drive the rearward flow of actin filaments? How does monomeric actin incorporate into the barbed ends of actin filaments that are closely associated with the plasma membrane? Are there any actin-depolymerizing factors localized at the base of growth cones and involved in the generation of the monomeric actin pool? These questions have yet to be answered, and further biochemical and structural studies on the molecular composition of the growth cone cytoskeleton will be required to provide more concrete information about these issues.

\section{References}

Abercrombie M, Heaysman JEM, Pegrum SM (1970) The locomotion of fibroblasts in culture. 2. Ruffling. Exp Cell Res 60:437-444.

Bamburg JR, Bray D (1987) Distribution and cellular localization of actin depolymerizing factor. J Cell Biol 105:2817-2825.

Bray D (1987) Growth cones: do they pull or are they pushed? Trends Neurosci 10:431-434.

Bray D, Chapman K (1985) Analysis of microspike movements on the neuronal growth cone. J Neurosci 5:3204-3213.

Bridgman PC, Dailey ME (1989) The organization of myosin and actin in rapid frozen nerve growth cones. J Cell Biol 108:95-109.

Dennerll TJ, Joshi HC, Steel VL, Buxbaum RE, Heidemann SR (1988) Tension and compression in the cytoskeleton of PC-12 neurites. 2: quantitative measurements. J Cell Biol 107:665-674.

Fisher GW, Conrad PA, DeBiasio RL, Taylor DL (1988) Centripetal transport of cytoplasm, actin, and the cell surface in lamellipodia of fibroblasts. Cell Motil Cytoskel 11:235-247.

Forscher P, Smith SJ (1988) Actions of cytochalasins on the organization of actin filaments and microtubules in a neuronal growth cone. J Cell Biol 107:1505-1516.

Forscher P, Kaczmarek LK, Buchanan J, Smith SJ (1987) Cyclic AMP induces changes in distribution of organelles within growth cones of Aplysia bag cell neurons. J Neurosci 7:3600-3611.

Goldenberg SSS, De Boni U (1983) Pure population of viable neurons from rabbit dorsal root ganglia, using gradients of Percoll. J Neurobiol 14:195-206.

Goodman CS, Bastiani MJ, Paper JA, Thomas JB (1985) Cell recognition during neuronal development in grasshopper and Drosophila. In: Molecular basis of neural development (Edelman GM, Gall WE, Cowen WM, eds), pp 295-316. New York: Neuroscience Research Foundation.

Gunderson RW, Barrett JN (1980) Characterization of the turning response of dorsal root neurites toward nerve growth factor. J Cell Biol 87:546-554.

Hamaguchi Y, Toriyama M, Sakai H, Hiramoto Y (1987) Redistribution of fluorescently labeled tubulin in the mitotic apparatus of sand dollar eggs and the effects of taxol. Cell Struct Funct 12:43-52.

Harris AK, Dunn GA (1972) Centripetal transport of attached particles on both surfaces of moving fibroblasts. Exp Cell Res 73:519-523.

Hatanaka H (1981) Nerve growth factor-mediated stimulation of tyrosine hydroxylase activity in a clonal rat pheochromocytoma cell line. Brain Res 222:225-233.

Heath JP (1981) Arcs: curved microfilament bundles beneath the dorsal surface of the leading lamellae of moving chick embryo fibroblasts. Cell Biol Int Rep 5:975-981.

Hirokawa N (1982) The crosslinker system between neurofilaments, microtubules and membranous organelles in frog axons revealed by 
quick-freeze, freeze-fracture, deep-etching method. J Cell Biol 94: $425-443$.

Hirokawa N (1991) Molecular architecture and dynamics of the neuronal cytoskeleton. In: Neuronal cytoskeleton (Burgoyne RD, ed), pp 5-74. New York: Liss.

Hirokawa N, Bloom GS, Vallee RB (1985) Cytoskeletal architecture and immunoelectrochemical localization of microtubule-associated proteins in regions of axons associated with rapid axonal transport: the IDPN-intoxicated axon as a model system. J Cell Biol 101:227239.

Kater S, Letourneau P (1985) Biology of the nerve growth cone. New York: Liss.

Kellog DR, Mitchison TJ, Alberts BM (1988) Behavior of microtubules and actin filaments in living Drosophila embryos. Development 103:675-686.

Korn ED (1982) Actin polymerization and its regulation by proteins from non-muscle cells. Physiol Rev 62:672-737.

Kreis TE, Winterhalter KH, Birchmeier W (1979) In vivo distribution and turnover of fluorescently labeled actin microinjected into human fibroblasts. Proc Natl Acad Sci USA 76:3814-3818.

Laemmli UK (1970) Cleavage of structural proteins during the assembly of the head of bacteriophage T4. Nature 227:680-685.

Lamoureux P, Buxbaum RE, Heidemann SR (1989) Direct evidence that growth cones pull. Nature 340:159-162.

Letourneau PC (1983) Differences in the organization of actin in the growth cones compared with the neurites of cultured neurons from chick embryos. J Cell Biol 97:963-972.

Lim SS, Sammak PJ, Borisy GG (1989) Progressive and spatially differentiated stability of microtubules in developing neuronal cells. J Cell Biol 109:253-263.

Lim SS, Edson KJ, Letourneau PC, Borisy GG (1990) A test of microtubule translocation during neurite elongation. J Cell Biol 111: 123-130.

Marsh L, Letourneau PC (1984) Growth of neurites without filopodial or lamellipodial activity in the presence of cytochalasin B. J Cell Biol 99:2041-2047.

Matus A (1988) Microtubule-associated proteins: their potential role in determining neuronal morphology. Annu Rev Neurosci 11:29-44.
Mitchison T, Kirschner M (1988) Cytoskeletal dynamics and nerve growth. Neuron 1:761-772.

Nakai J (1979) The movement of the neuron-elongation and retraction of nerve fibers and filopodia. In: Cell motility: molecules and organization (Hatano S, Ishikawa $\mathrm{H}$, Sato H, eds), pp 263-272. Tokyo: University of Tokyo.

Nakai J, Kawasaki Y (1960) Studies on the mechanism determining the course of nerve fibers in tissue culture. Z Zellforsch Mikrosk Anat 51:108-122.

Okabe S, Hirokawa N (1988) Microtubule dynamics in nerve cells: analysis using microinjection of biotinylated tubulin into $\mathrm{PC} 12$ cells. J Cell Biol 107:651-664.

Okabe S, Hirokawa N (1989a) Rapid turnover of microtubule-associated protein MAP2 in the axon revealed by microinjection of biotinylated MAP2 into cultured neurons. Proc Natl Acad Sci USA 86: $4127-4131$.

Okabe S, Hirokawa N (1989b) Incorporation and turnover of biotinlabeled actin microinjected into fibroblastic cells: an immunoelectron microscopic study. J Cell Biol 109:1581-1595.

Okabe S, Hirokawa N (1990) Turnover of fluorescently labelled tubulin and actin in the axon. Nature 343:479-482.

Rogers SL, Letourneau PC, Palm SL, McCarthy J, Furcht LT (1983) Neurite extension by peripheral and central nervous system neurons in response to substratum-bound fibronectin and laminin. Dev Biol 98:212-220.

Sheetz MP, Turney S, Qian H, Elson EL (1989) Nanometre-level analysis demonstrates that lipid flow does not drive membrane glycoprotein movements. Nature 340:284-288.

Small JV, Isenberg G, Celis JE (1978) Polarity of actin at the leading edge of cultured cells. Nature 272:638-639.

Smith SJ (1988) Neuronal cytomechanics: the actin-based motility of growth cones. Science 242:708-715.

Taylor DL, Wang Y-L (1978) Molecular cytochemistry: incorporation of fluorescently labeled actin into living cells. Proc Natl Acad Sci USA 75:857-861.

Wang Y-L (1985) Exchange of actin subunits at the leading edge of living fibroblasts: possible role of treadmilling. J Cell Biol 101:597602 . 